\title{
An interacting segment model of molecular electric tensor properties: Theory and application to electric dipole moments of the halogenated methanes ${ }^{\text {a) }}$
}

\author{
C. K. Miller, B. J. Orr, ${ }^{\text {b) }}$ and J. F. Ward \\ Randall Laboratory of Physics. The University of Michigan, Ann Arbor, Michigan 48109 \\ (Received 4 May 1977) \\ A model is developed to describe trends in molecular properties such as electric dipole moment, \\ polarizabilities, and hyperpolarizabilities for series of structurally related molecules. The model takes \\ account of intramolecular electrostatic interactions, which give rise to induced electric moments in the \\ various segments of the molecule. For this purpose each molecular segment is ascribed a set of "bare" \\ electric tensor properties, which describe the hypothetical situation where intersegment interactions are \\ absent, and a corresponding set of "dressed" properties, which include the effects of the intramolecular \\ field. Any overall molecular electric tensor property is then obtained by summing the appropriate dressed \\ segment properties. After theoretical development the model is used to fit electric dipole moment data for \\ the 12 halogenated methanes $\mathrm{CX}_{n} \mathrm{Y}_{4-n}(\mathrm{X}, \mathrm{Y}=\mathrm{H}, \mathrm{F}, \mathrm{Cl} ; n=0-4)$, for which a simple bond additivity \\ model is known to be inadequate and which severely test any theory of induced dipole moments. The \\ results of such an analysis are highly satisfactory and indicate that the model should also give an adequate \\ description of higher-order electric tensor properties.
}

\section{INTRODUCTION}

Many significant molecular processes depend on the interaction between a molecule and a uniform electric field, and can conveniently be described by expanding the total electric dipole moment $\mu(\mathbb{E})$ in powers of the electric field strength $\mathbf{E}$ :

$$
\boldsymbol{\mu}(\mathbf{E})=\boldsymbol{\mu}+\boldsymbol{\alpha} \mathbf{E}+\zeta \boldsymbol{\beta} \mathbf{E}^{2}+\lambda \boldsymbol{\gamma} \mathbf{E}^{3}+\cdots .
$$

The leading term $\mu$ is the permanent electric dipole moment of the molecule in the absence of the field $\mathbf{E}$ and subsequent terms involve the molecular polarizability tensor $\alpha$ and molecular hyperpolarizability tensors $\beta$, $\gamma, \ldots{ }^{1}$ It suffices in the present discussion to regard $\mathbf{E}$ as a dc field, in which case $\zeta, \lambda, \ldots$ are numbers of order unity whose assignment depends on convention. ${ }^{2}$ Extension to the case of time-dependent fields is straightforward, involving only the definition of $\mathbf{E}$ as a field amplitude and reassignment of the values of $\zeta, \lambda, \ldots$ These details are set out in the Appendix.

Experimental data on the molecular electric properties $\mu, \alpha, \beta$, and $\gamma$ are now available from a wide range of sources, ${ }^{1}$ and permit comparisons to be made from molecule to molecule. For simple molecules, such as those of the first-row elements, $a b$ initio quantum mechanical calculations are able to predict the properties $\mu$ and $\alpha$ and are generally consistent with corresponding experimental data. The few ab initio computations of molecular $\beta$ and $\gamma$ data which exist yield only order-of-magnitude agreement with experiment. As molecules increase in size and complexity accurate $a b$ initio calculations become increasingly difficult, so that it is customary to analyze observed results for such molecules in terms of semiempirical correlations with other chemically and physically similar molecules. The most widely used correlation scheme is the bond ad-

a) Research supported in part by the Air Force Office of Scientific Research under Grants Nos. 72-2302 and 77-3225.

b)Permanent address: School of Chemistry, University of New South Wales, Kensington, N. S. W. , Australia 2033. ditivity approximation (BAA), in which a molecule is subdivided into a number of segments $i$, corresponding to its individual bonds and/or functional groups, and a given molecular tensor property $\chi$ is assumed to be the sum of its corresponding segment tensors $\chi_{i}$ :

$$
x=\sum_{i} \chi_{i} .
$$

The application of such an approximation to molecular electric properties has a long history, particularly in relation to electric dipole moments $\mu$ and to the isotropic part $\alpha$ of the molecular polarizability tensor. In the former case the fact that Eq. (2) is a vectorial sum of segment electric dipole moments $\mu_{i}$ has enabled electric dipole moment determinations to provide useful information about molecular geometries. ${ }^{3}$ In the latter case $\alpha$ conforms closely to scalar bond additivity laws within a given homologous series of molecules and is recognized as a characteristic molecular property which is independent of structural geometry. ${ }^{4,5}$ The BAA has also been applied to the anisotropic part of the polarizability $\alpha$ and extensively employed in studies of molecular structure. ${ }^{5}$ More recently versions of the BAA have been proposed ${ }^{6}$ and applied ${ }^{7-10}$ with mixed success to the hyperpolarizabilities $\beta$ and $\gamma$, which can be obtained ${ }^{2}$ from various nonlinear optical experiments.

The conceptual division of a molecule into individual segments, which is the basis for the BAA, is inherent in discussions of such accepted and useful quantities as bond energies, group vibrational frequencies, and molecular geometric parameters. For the BAA to be valid for electric tensor properties it is necessary that characteristic electric tensors can be assigned to individual segments and that these should not be modified by interactions with neighboring segments. There is no a priori justification for the assumption of negligible electrostatic intersegment interaction, as can be demonstrated by a simple calculation. Consider, for example, the electric dipole moment $\Delta \mu_{\mathrm{A}}$ induced in a molecular segment $A$ by its interaction with an adjacent segment $B$ at distance $R$. The electric field at A due to the electric 
dipole moment $\mu_{B}$ of segment $\mathrm{B}$ is approximately $\mu_{\mathrm{B}} R^{-3}$, so that the induced moment $\Delta \mu_{\mathrm{A}}$ is of order $\alpha_{\mathrm{A}} \mu_{\mathrm{B}} R^{-3}$, where $\alpha_{\mathrm{A}}$ is the polarizability (assumed isotropic) of segment $\mathrm{A}$. Both $\alpha_{\mathrm{A}}$ and $R^{3}$ are measures of a molecular bond or group volume and their ratio is of order unity, so that the induced moment $\Delta \mu_{A}$ is comparable in magnitude to its source $\mu_{B}$. Attempts to assess the role of such inductive effects in determining molecular electric dipole moments have been reviewed by Smith. ${ }^{3}$ Several workers ${ }^{11-15}$ have indicated the consequences of neglecting interactions between segments within a molecule, with regard to molecular polarizability. It is a feature of any treatment of intramolecular electrostatic interactions that $\alpha R^{-3}$, the parameter which scales the effect of such interactions, is comparable in magnitude to unity and that the interactions are therefore not in general negligible.

In view of the above it is remarkable that simple BAA schemes have achieved their generally accepted level of success in treating electric dipole moment and polarizability data. The early criticism of Pitzer ${ }^{11}$ that "some fortuitous cancellation of conflicting factors" probably accounts for the applicability of the BAA to a limited range of molecules, remains valid. Such a situation is indeed suggested by the need to assign, within the framework of the BAA, different bond and group polarizabilities for markedly different molecular environments ${ }^{5}$ and by the inadequacy of the simple BAA in treating the electric dipole moments of such molecules as those in the halogenated methane series. ${ }^{3}$ It would appear that empirical electric tensor properties for a given species of segment, deduced from experimental data for a series of molecules, must contain, in addition to the intrinsic segment contribution, a substantial but undetermined contribution arising from interactions between segments.

There is some recent theoretical support for the proposition that individual bond contributions to molecular electric dipole moments ${ }^{16,17}$ and polarizabilities ${ }^{18}$ may in certain instances be identified and transferred from molecule to molecule. These studies are based on localized molecular orbital theory and are confined to the contributions of $\mu(\mathrm{C}-\mathrm{H}), \alpha(\mathrm{C}-\mathrm{H})$, and $\alpha(\mathrm{C}-\mathrm{C})$ in simple hydrocarbon molecules.

A further factor which may assist the apparent validity of the BAA in certain circumstances arises from local field effects in condensed phases. Discrepancies have recently been noted ${ }^{15}$ between the gas- and liquid-phase results obtained from light scattering or electric birefringence measurements of simple alkanes. The discrepancies have been attributed ${ }^{15}$ to the more symmetrical environment of a molecular segment when it is surrounded by a random distribution of nearby molecules in the condensed phase, compared to the relatively anisotropic environment of the segment in an isolated (gasphase) molecule. A similar argument ${ }^{19}$ might explain why a simple BAA scheme appears to be much more successful in fitting hyperpolarizability data obtained in condensed phase ${ }^{10}$ than in fitting those determined for isolated molecules in the gas phase. ${ }^{7-\theta}$ Our present concern is with the understanding of electric dipole

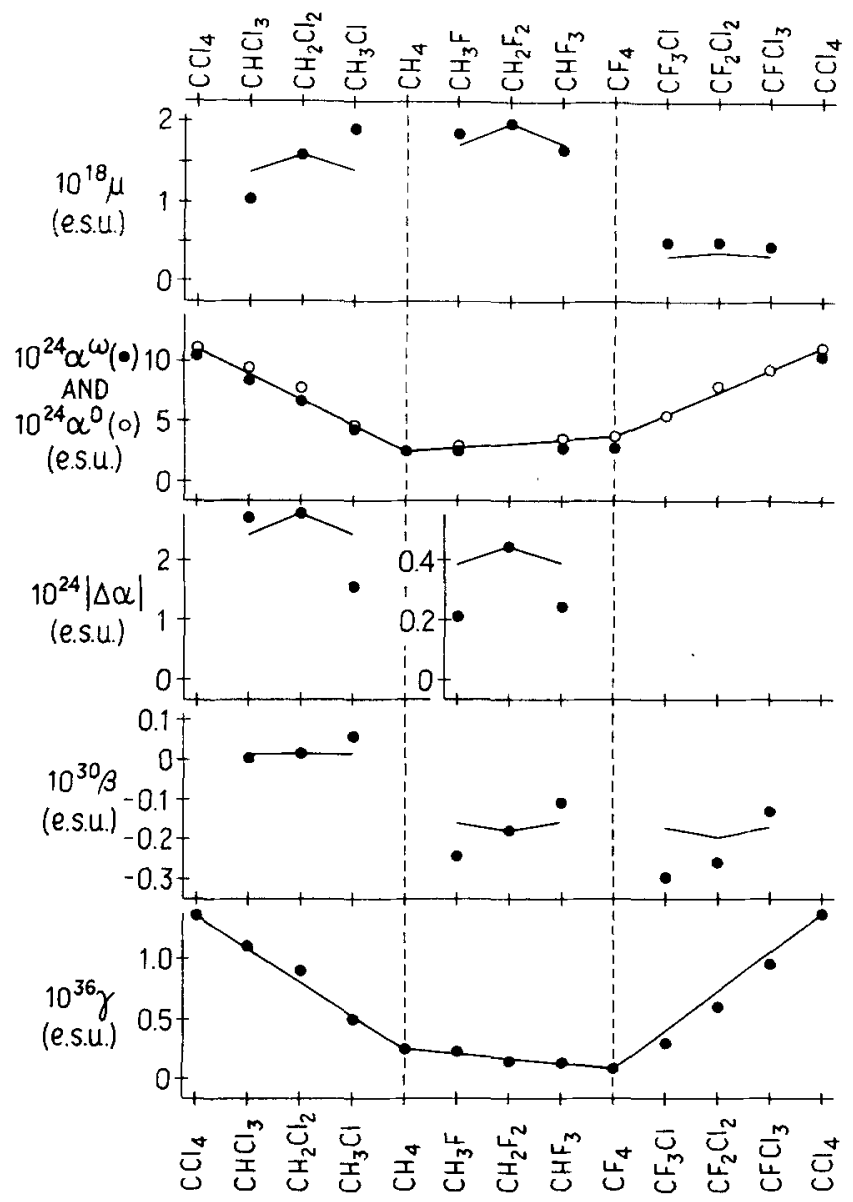

FIG. 1. Electric dipole properties of the halogenated methanes. The definitions of the molecular observables, $\mu, \alpha^{\omega}, \alpha^{0}, \Delta \alpha$, $\beta$, and $\gamma$ are given in the Appendix and sources of data are indicated in the text. The solid lines are fitted by application of the simple bond additivity approximation (BAA) to the data for $\mathrm{CH}_{2} \mathrm{X}_{2}$ (in the case of $\mu,|\Delta \alpha|$, and $\beta$ ) and $\mathrm{CX}_{4}$ molecules (in the case of $\alpha^{0}$ and $\gamma$ ), with the assumption of tetrahedral bond angles.

properties of isolated molecules, so that in the discussion which follows only gas-phase experimental data are considered.

We shall concentrate attention on the halogenated derivatives of methane, which have provided a severe test of semiempirical correlation schemes for electric dipole moments $\mu$ and, more recently, for polarizabilities $\alpha$ and hyperpolarizabilities $\beta$ and $\gamma$. The understanding of trends in the electric dipole moments within this series of molecules has advanced relatively little since the classic work of Smyth and McAlpine ${ }^{20}$ in 1933, in which approximate account was taken of mutual inductive effects between $\mathrm{C}-\mathrm{H}, \mathrm{C}-\mathrm{F}$, and $\mathrm{C}-\mathrm{Cl}$ bond dipole moments. Of subsequent developments ${ }^{3}$ the best fit to experimental electric dipole moments of the halogenated methanes appears to have been achieved by a model ${ }^{21}$ which treats the inductive effect semiclassically in terms of screened nuclear charges. Although this model proves consistent with dipole moment data for molecules $\mathrm{CH}_{n} \mathrm{X}_{4-n}(\mathrm{X}=$ halogen), it is inadequate for molecules such as $\mathrm{CF}_{n} \mathrm{Cl}_{4-n}$, in which inductive contributions are known ${ }^{20}$ to be dominant. In Fig. 1 we summarize the molecular observables of a number of electric dipole 
tensor properties, for 12 chloro and fluoro derivatives of methane. The observables and their sources of determination ${ }^{22}$ are the permanent electric dipole moment $\mu$ from microwave spectra and dielectric constant data, ${ }^{23,24}$ the mean optical polarizability $\alpha^{\omega}$ determined from refractivity data for $\lambda \sim 640 \mathrm{~nm},{ }^{25-27}$ the mean static polarizability $\alpha^{0}$ from dielectric constant data, $1,28-30$ the polarizability anisotropy parameter ${ }^{22}|\Delta \alpha|=3 \alpha^{\omega}|\kappa|$ from studies of depolarization of Rayleigh scattering ${ }^{31}$ and from the temperature dependence of the electric birefringence, ${ }^{7}$ and the hyperpolarizabilities ${ }^{22} \beta$ and $\gamma$ from dc-induced second harmonic generation studies. ${ }^{9,32}$ Also shown in Fig. 1 are lines indicating the application of a simple BAA correlation scheme. For the isotropic properties $\alpha^{\omega}, \alpha^{0}$, and $\gamma$ the BAA predicts a linear relationship in the number of substituents, whereas anisotropic properties such as $\mu,|\Delta \alpha|$, or $\beta(=\chi$, say) would be expected to fit relationships of the type $\chi\left(\mathrm{CX}_{3} \mathrm{Y}\right)$ $=\chi\left(\mathrm{CXY}_{3}\right)=\sqrt{3} / 2 \chi\left(\mathrm{CX}_{2} \mathrm{Y}_{2}\right)$, assuming tetrahedral valence angles. It is evident that $\alpha^{\omega}$ and $\alpha^{0}$ appear remarkably consistent with the BAA, and that the trends in $\gamma$ are also well represented by the BAA. The anisotropic properties $\mu,|\Delta \alpha|$, and $\beta$, on the other hand, are noteworthy for their marked inconsistency with any simple BAA relationship. In fact, the BAA is hardly any better in these cases than a crude dimensional argument, which would predict that a given observable should be of the same order of magnitude within a set of molecules.

In this paper we develop an interacting-segment model (ISM) to seek better agreement with data such as those displayed in Fig. 1, without completely abandoning the simplicity of the BAA. The theoretical framework of the ISM is presented in Sec. II, and in Sec. III the model is applied in detail to the electric dipole moments of the halogenated methanes. A full-scale application of the ISM to all of the properties $\mu, \alpha, \Delta \alpha, \beta$, and $\gamma$ shown in Fig. 1 will be reported in a subsequent paper. ${ }^{33} \mathrm{~A}$ simplified version of the ISM has also been used to provide a preliminary analysis of the hyperpolarizabilities $\beta$ of the halogenated methanes. ${ }^{32}$

Very recently, Sundberg ${ }^{34}$ has developed a group-dipole interaction model which is formally similar to our ISM.

\section{THEORY}

We consider a molecular model comprising a number of segments, corresponding to the individual bonds and/ or functional groups of the molecule. In the absence of intersegment interactions we suppose that the segment $i$ can be characterized by a set of "bare" segment parameters $\mu_{i}, \alpha_{i}, \beta_{i}, \gamma_{i}, \ldots$; these are tensor properties which conform to the symmetry of the isolated segment, so that a heteronuclear $\sigma$ bond would have undressed tensor properties with $C_{\infty}$ symmetry about the bond axis. When account is taken of intersegment interactions the same segment is characterized by a different set of parameters $\hat{\mu}_{i}, \hat{\alpha}_{i}, \hat{\beta}_{i}, \hat{\gamma}_{i}, \ldots$ which are "dressed" by electrostatic interactions with neighboring segments of the molecule; the dressed tensors conform in general to a lower symmetry than that of the undressed segment, in view of the anisotropy of the intra- molecular interactions. The resultant molecular tensor property $\chi$ will then be given by the sum over segments of the corresponding dressed segment tensors $\hat{\chi}_{i}$.

The electric dipole moment of the $i$ th segment in the presence of a uniform applied electric field $\mathbf{E}$ may be written in terms of the dressed tensors and the applied field:

$$
\begin{aligned}
\mu_{i}(\mathbf{E}) & =\hat{\mu}_{i}+\hat{\alpha}_{i} \mathbf{E}+\hat{\beta}_{i} \mathbf{E}^{2}+\hat{\gamma}_{i} \mathbf{E}^{3}+\cdots \\
& =\sum_{n=0}^{\infty} \hat{\chi}_{i}^{(n)} \mathbf{E}^{n} .
\end{aligned}
$$

This equation is the same as Eq. (1) except that here it is applied to individual segments rather than the complete molecule, and $\zeta, \lambda$ have been set equal to unity. This choice of $\zeta$ and $\lambda$ and the relevance of Eq. (3) to the case where time varying fields are present is discussed in the Appendix. $\hat{\chi}_{i}^{(n)}$ is defined by Eq. (3) to be $\hat{\mu}_{i}, \hat{\alpha}_{i}$, $\hat{\boldsymbol{\beta}}_{i}, \hat{\gamma}_{i}, \ldots$ for $n=0,1,2,3, \ldots$, respectively. $\mu_{i}(\mathbf{E})$ can equally well be expressed in terms of the total electric field $\mathbf{E}_{i}$ which is the sum of the external applied field $\mathbf{E}$ and fields produced at segment $i$ by polarized neighboring segments. This expression involves the bare parameters of the $i$ th segment and is

$$
\begin{aligned}
\mu_{i}(\mathbf{E}) & =\mu_{i}+\alpha_{i} \mathbf{E}_{i}+\beta_{i} \mathbf{E}_{i}^{2}+\gamma_{i} \mathbf{E}_{i}^{3}+\cdots \\
& =\sum_{n=0}^{\infty} \chi_{i}^{(n)} \mathbf{E}_{i}^{n} .
\end{aligned}
$$

In Eqs. (3) and (4) we ignore nonclassical short-range interactions, of the type known ${ }^{35}$ to be significant at distances as short as those between segments in a typical molecule, and assume intersegment interactions to be electrostatic in origin. In order more simply to evaluate $\mathbf{E}_{i}$ we will further assume that all segment tensors are localized within the segment at a single point, which we call the "active center," and that contributions from segment electric multipoles of order higher than dipole can be neglected. Such drastic approximations must, of course, be justified by the ability of the model to reproduce molecular observables and to provide a set of empirical undressed parameters $\mu_{i}, \alpha_{i}, \beta_{i}, \gamma_{i}, \ldots$ which are transferable, for a given species of bond or functional group, from one molecule to another. With these approximations the electric field $F^{(i j)}$ induced at the active center of segment $i$ due to the dressed point electric dipole $\hat{\mu}_{j}$ at the active center of a neighboring segment $j$ is $T_{i f} \hat{\mu}_{j}$ or, more explicitly, ${ }^{36,37}$

$$
F_{!}^{(i j)}=T_{i j ! n} \hat{\mu}_{j \eta},
$$

where

$$
\begin{aligned}
T_{i j \xi \eta}=T_{j i \xi \eta} & =\left(3 R_{i j \xi} R_{i j \eta}-R_{i j}^{2} \delta_{\xi \eta}\right) R_{i j}^{-5}, j \neq i \\
& =0, \quad j=i,
\end{aligned}
$$

and $R_{i j}$ is the distance vector between the active centers of segments $i$ and $j$.

The total electric field $\mathbf{E}_{i}$ at a segment $i$, resulting from the application of a uniform electric field $\mathbf{E}$ external to the molecule, may now be written as

$$
\mathbf{E}_{i}=\mathbf{E}+\sum_{j \neq i} \mathbf{T}_{i j} \mu_{j}(\mathbf{E}) .
$$


Combination with Eq. (3) yields

$$
\mathbf{E}_{i}=\mathbf{E}+\sum_{j \neq i} \mathrm{~T}_{i j} \sum_{n=0}^{\infty} \hat{\chi}_{j}^{(n)} \mathrm{E}^{n}
$$

Substitution of this expression for $\mathrm{E}_{i}$ into $\mathrm{Eq}$. (4) gives a polynomial in $\mathbf{E}$ which can be compared term by term with Eq. (3) to yield the relations between the dressed and bare quantities which we seek. Equivalently, Eq.

(8) can be used together with

$$
\hat{\chi}_{i}^{(m)}=\sum_{n=0}^{\infty} \chi_{i}^{(n)} \operatorname{Lim}_{\mathbf{E} \rightarrow 0}\left[\partial^{m}\left(\mathbf{E}_{i}^{n}\right) / \partial(\mathbf{E})^{m}\right] / m !,
$$

which follows from Eqs. (3) and (4). Introducing an abbreviated notation ${ }^{38}$ the results are

$$
\begin{aligned}
\hat{\mu}_{i}= & \mu_{i}+\alpha_{i}\langle\mathbf{T} \cdot \hat{\mu}\rangle_{i}+\beta_{i}\langle\mathbf{T} \cdot \hat{\mu}\rangle_{i}^{2}+\gamma_{i}\langle\mathbf{T} \cdot \hat{\mu}\rangle_{i}^{3}+\ldots, \\
\hat{\alpha}_{i}= & \left(\alpha_{i}+2 \beta_{i}\langle\mathbf{T} \cdot \hat{\mu}\rangle_{i}+3 \gamma_{i}\langle\mathbf{T} \cdot \hat{\mu}\rangle_{i}^{2}+\ldots\right)\left(1+\langle\mathbf{T} \cdot \hat{\alpha}\rangle_{i}\right), \\
\hat{\beta}_{i}= & \alpha_{i}\langle\mathbf{T} \cdot \hat{\beta}\rangle_{i}+\beta_{i}\left[2\langle\mathbf{T} \cdot \hat{\mu}\rangle_{i}\langle\mathbf{T} \cdot \hat{\beta}\rangle_{i}+\left(1+\langle\mathbf{T} \cdot \hat{\alpha}\rangle_{i}\right)^{2}\right] \\
& +3 \gamma_{i}\langle\mathbf{T} \cdot \hat{\mu}\rangle_{i}\left[\langle\mathbf{T} \cdot \hat{\mu}\rangle_{i}\langle\mathbf{T} \cdot \hat{\beta}\rangle_{i}+\left(1+\langle\mathbf{T} \cdot \hat{\alpha}\rangle_{i}\right)^{2}\right]+\ldots, \\
\hat{\gamma}_{i}= & \alpha_{i}\langle\mathbf{T} \cdot \hat{\gamma}\rangle_{i}+2 \beta_{i}\left[\langle\mathbf{T} \cdot \hat{\mu}\rangle_{i}\langle\mathbf{T} \cdot \hat{\gamma}\rangle_{i}+\left(1+\langle\mathbf{T} \cdot \hat{\alpha}\rangle_{i}\right)\langle\mathbf{T} \cdot \hat{\beta}\rangle_{i}\right] \\
& +\gamma_{i}\left[3\langle\mathbf{T} \cdot \hat{\mu}\rangle_{i}^{2}\langle\mathbf{T} \cdot \hat{\gamma}\rangle_{i}+6\langle\mathbf{T} \cdot \hat{\mu}\rangle_{i}\left(1+\langle\mathbf{T} \cdot \hat{\alpha}\rangle_{i}\right)\langle\mathbf{T} \cdot \hat{\beta}\rangle_{i}\right. \\
& \left.+\left(1+\langle\mathbf{T} \cdot \hat{\alpha}\rangle_{i}\right)^{3}\right]+\ldots .
\end{aligned}
$$

The only contributions which are not specified in Eqs. (10)-(13) are those which would arise from terms in Eqs. (7) and (8) involving $\hat{\chi}_{i}^{(n)}$ and $\chi_{i}^{(n)}$ with $n>3$, i. e., the above equations are complete through the order of hyperpolarizabilities $\hat{\gamma}_{i}$ and $\gamma_{i}$.

The above set of coupled equations forms the basis for attempts to determine self-consistent sets of dressed and undressed electric tensors from appropriate molecular observables. To achieve this it is desirable to rearrange the equations so that the expression for a given dressed tensor $\hat{\chi}_{i}^{(m)}$ is not functionally dependent on terms which contain $\left\langle\mathbf{T} \cdot \hat{\boldsymbol{X}}^{(m)}\right\rangle_{i}$. In the present paper we consider this problem for the electric dipole moment $\hat{\mu}_{i}$. The corresponding situations for $\hat{\alpha}_{i}, \hat{\beta}_{i}$, and $\hat{\gamma}_{i}$ will be dealt with in a subsequent paper. ${ }^{33}$

Equation (10) may be rearranged as follows:

$\sum_{j}\left\{\alpha_{i}^{-1} \delta_{i j}-\mathrm{T}_{i j}\right\} \hat{\mu}_{j}=\alpha_{i}^{-1}\left(\mu_{i}+\beta_{i}\langle\mathbf{T} \cdot \hat{\mu}\rangle_{i}^{2}+\gamma_{i}\langle\mathrm{~T} \cdot \hat{\mu}\rangle_{i}^{3}\right)$.

An expression for $\hat{\mu}_{i}$ is then obtained by forming the inverse $\mathrm{M}$ of the matrix quantity $\{\cdots\}$ in Eq. (14), in the manner exploited in polarizability calculations by Applequist et $a l .^{14,39}$

$$
\hat{\mu}_{i}=\sum_{j} \mathbf{M}_{i j} \alpha_{j}^{-1}\left(\mu_{j}+\beta_{j}\langle\mathbf{T} \cdot \hat{\mu}\rangle_{j}^{2}+\gamma_{j}\langle\mathbf{T} \cdot \hat{\mu}\rangle_{j}^{3}\right)
$$

The significance of $\mathbf{M}$ can be demonstrated by manipulating Eq. (11) with $\beta_{i}, \gamma_{i}=0$ to yield

$$
\sum_{j} \mathbf{M}_{i j}=\hat{\alpha}_{i} .
$$

This is identical to the result of Applequist et al. ${ }^{14}$ Further manipulation of Eq. (16) also reproduces the results of the Silberstein model, ${ }^{40,41}$ which first demonstrated how intramolecular electrostatic interactions can produce an anisotropic polarizability tensor for a molecule composed of isotropically polarizable atomic segments.

The previously stated objective, of casting the expression for $\hat{\boldsymbol{\mu}}_{i}$ in a form which is independent of terms containing $\langle\mathbf{T} \cdot \hat{\boldsymbol{\mu}}\rangle_{i}$, has not been satisfied by Eq. (15), since $\hat{\mu}_{i}$ is a nonlinear function of $\langle\mathrm{T} \cdot \hat{\mu}\rangle_{j}$. However, Eq. (15) does provide a suitable basis for computations of $\hat{\mu}_{i}$ and details of its application will be considered in the next section.

\section{APPLICATION TO THE DIPOLE MOMENTS OF SOME HALOGENATED METHANES}

We wish to understand the measured dipole moments of nine halogenated-methane molecules (a list is given in Table III) in terms of the interacting segment model (ISM) as expressed by Eq. (15). The procedure may be summarized as follows: (1) Select a set of parameters $\mathbf{T}_{i j}$ which describe the interaction geometry for each pair of bonds in each molecule; (2) select electric tensor parameters $\alpha_{i}, \beta_{i}, \gamma_{i}$ for each type of bond; and (3) compute values of bare bond moments $\mu_{i}$ which, using Eq. (15), yield values for molecular dipole moments $\mu^{\prime}$ that best fit the experimental molecular moments $\mu$ for the set of molecules considered. The steps in this procedure will now be considered in more detail before presenting the results.

A reference set of parameters is shown in Table $I$. Since a simultaneous fit to all hyperpolarizability data is not being attempted at this stage, it has been necessary to estimate bare bond parameters other than $\mu_{i}$. The nature of these estimates is indicated in the table. The choice of active center locations $\mathbf{r}_{i}$ forms part of the statement of the model. Rather than adjusting these parameters for best fit we elect to use a specific, arbitrary set of locations consistent with intuition and similar to those selected by Smyth and McAlpine. ${ }^{20}$ Other reasonable choices of the $r_{i}$ lead to qualitatively similar results. The effect of using bond angles and polarizability anisotropies differing from the reference set will be discussed briefly later.

Dimensional arguments discussed in Sec. I. lead to the conclusion that terms $\mu_{i}, \beta_{i}\langle\mathbf{T} \cdot \hat{\mu}\rangle_{i}^{2}$, and $\gamma_{i}\langle\mathbf{T} \cdot \hat{\mu}\rangle_{i}^{3}$ appearing in Eq. (15) are all of comparable magnitude. However, we adopt the hypothesis that $\mu_{i}$ and $\boldsymbol{\beta}_{i}$, which vanish in centrosymmetric systems, are overestimated by the dimensional arguments so that $\beta_{i}\langle\mathrm{~T} \cdot \hat{\mu}\rangle_{i}^{2}$ and $\gamma_{i}\langle\mathbf{T} \cdot \hat{\mu}\rangle_{i}^{3}$ may both be smaller than $\mu_{i}$. Any cancellation within the vector sum over bonds $\langle\mathbf{T} \cdot \hat{\mu}\rangle_{i}$ will reinforce this tendency. This hypothesis allows an iterative solution of Eq. (15) which yields, as the result of the $p$ th iteration, the bare and dressed bond moments $\mu_{i}^{(p)}$ and $\hat{\mu}_{i}^{(b)}$, respectively. The procedure is described by the sequence of Eqs. (17)-(20), where the steps indicated by Eqs. (18) and (19) are carried out iteratively

$\hat{\mu}_{i}^{(0)}=0$,

$\mu-\sum_{i, j} \mathbf{M}_{i j} \alpha_{j}^{-1}\left(\beta_{j}\left\langle\mathbf{T} \cdot \hat{\mu}^{(p-1)}\right\rangle_{j}^{2}\right.$

$\left.+\gamma_{j}\left\langle\mathbf{T} \cdot \hat{\mu}^{(p-1)}\right\rangle_{j}^{3}\right)=\sum_{i, j} \mathbf{M}_{i j} \alpha_{j}^{-1} \mu_{j}^{(p)}$. 
TABLE I. Reference set of bond and structural parameter values for the halogenated methanes. ${ }^{2}$

\begin{tabular}{|c|c|c|c|c|}
\hline \multirow[b]{2}{*}{ Parameter } & \multicolumn{3}{|c|}{$\mathrm{C}-\mathrm{X}$ bond } & \multirow[b]{2}{*}{ Comments } \\
\hline & $\mathrm{C}-\mathrm{F}$ & $\mathrm{C}-\mathrm{Cl}$ & $\mathrm{C}-\mathrm{H}$ & \\
\hline $10^{24} \alpha(\mathrm{C}-\mathrm{X}) / \mathrm{cm}^{3}$ & 0.96 & 2.8 & 0.65 & $\begin{array}{l}\text { The isotropic bare bond polarizability } \alpha(\mathrm{C}-\mathrm{X}) \text { is approxi- } \\
\text { mated by the dressed polarizability } \hat{\alpha}^{0}(\mathrm{C}-\mathrm{X})=\frac{1}{4} \alpha^{0}\left(\mathrm{CX}_{4}\right) \\
\text { from Refs. } 29 \text { and } 1 .\end{array}$ \\
\hline $\begin{array}{l}10^{24}\left[\alpha_{11}-\alpha_{1}\right] \\
(\mathrm{C}-\mathrm{X}) / \mathrm{cm}^{3}\end{array}$ & 0.75 & 2.00 & 0.35 & $\begin{array}{l}\text { The bare bond polarizability anisotropy }\left(\alpha_{11}-\alpha_{1}\right)(\mathrm{C}-\mathrm{X}) \\
\text { is approximated by the dressed anisotropy } \\
\left(\hat{\alpha}_{11}^{0}-\hat{\alpha}_{1}^{0}\right)(\mathrm{C}-\mathrm{X})=\hat{\alpha}^{0}(\mathrm{C}-\mathrm{X})\left(\hat{\alpha}_{11}^{\omega}-\hat{\alpha}_{1}^{\omega}\right)(\mathrm{C}-\mathrm{X}) / \hat{\alpha}^{\omega}(\mathrm{C}-\mathrm{X}) \text {, where } \\
\hat{\alpha}^{\omega}(\mathrm{C}-\mathrm{X})=\frac{1}{4} \alpha^{\omega}\left(\mathrm{CX} \mathrm{X}_{4}\right) \text { from Refs. } 25-27 \text { and }\left(\hat{\alpha}_{\| 1}^{\omega}-\hat{\alpha}_{1}^{\omega}\right)(\mathrm{C}-\mathrm{X}) \\
\text { is determined crudely from a combination of theoreti- } \\
\text { cal }^{18} \text { and Raman intensity }{ }^{42} \text { data from molecules } \mathrm{CX} \\
\text {. }\end{array}$ \\
\hline $10^{30} \beta_{\|}(\mathrm{C}-\mathrm{X}) / \mathrm{esu}$ & +0.103 & +0.223 & +0.303 & $\beta_{\| 1}(\mathrm{C}-\mathrm{X})=\beta_{z z z}(\mathrm{C}-\mathrm{X})$ and $\beta_{1}(\mathrm{C}-\mathrm{X})=\beta_{x x z}(\mathrm{C}-\mathrm{X})=\beta_{y y z}(\mathrm{C}-\mathrm{X})$ \\
\hline $10^{30} \beta_{1}(\mathrm{C}-\mathrm{X}) / \mathrm{esu}$ & -0.127 & -0.265 & +0.104 & $\begin{array}{l}=\ldots \text { (where the bond } z \text { axis points from } \mathrm{X} \text { to } \mathrm{C} \text { ) are } \\
\text { determined from a preliminary fit of experimental } \\
\text { hyperpolarizabilities } \beta^{\text {sHG }} \text { to Eq. (12). }\end{array}$ \\
\hline $10^{36} \gamma(\mathrm{C}-\mathrm{X}) / \mathrm{esu}$ & 0.023 & 0.342 & 0.066 & $\begin{array}{l}\text { The bare bond hyperpolarizability } \gamma(\mathrm{C}-\mathrm{X}) \text { is approxi- } \\
\text { mated by the dressed hyperpolarizability } \hat{\gamma}^{\mathrm{ESHG}}(\mathrm{C}-\mathrm{X}) \\
=\frac{1}{4} \gamma^{\mathrm{ESHG}}\left(\mathrm{CX}_{4}\right) \text { from Ref. } 32 . \gamma(\mathrm{C}-\mathrm{X}) \text { is assumed to be } \\
\text { isotropic. }\end{array}$ \\
\hline $10^{8} R(\mathrm{C}-\mathrm{X}) / \mathrm{cm}$ & 1.36 & 1.77 & 1.10 & $\begin{array}{l}\text { Representative internuclear distances for } \mathrm{C}-\mathrm{X} \text { bonds in } \\
\text { the halogenated methane series. See Ref. } 43 \text {. }\end{array}$ \\
\hline$f$ & 1.0 & 1.0 & 0.5 & $\begin{array}{l}f=r(\mathrm{C}-\mathrm{X}) / R(\mathrm{C}-\mathrm{X}) \text { defines the fractional position of the } \\
\text { active center along the } \mathrm{C}-\mathrm{X} \text { bond. The choice of } f \text { is } \\
\text { discussed in the text. }\end{array}$ \\
\hline \multirow[t]{2}{*}{$\theta(\mathrm{X}-\mathrm{C}-\mathrm{X})$ in $\mathrm{CX}_{3} \mathrm{Y}$} & $\begin{array}{l}108.0^{\circ}, \mathrm{Y}=\mathrm{H} \\
108.6^{\circ}, \mathrm{Y}=\mathrm{Cl}\end{array}$ & $\begin{array}{l}110.9^{\circ}, Y=H \\
111.7^{\circ}, Y=F\end{array}$ & $\begin{array}{l}110.9^{\circ}, \mathrm{Y}=\mathrm{Cl} \\
110.1^{\circ}, \mathrm{Y}=\mathrm{F}\end{array}$ & \\
\hline & & & & $\begin{array}{l}\mathrm{X}-\mathrm{C}-\mathrm{X} \text { bond angles for the halogenated methane series } \\
\text { from Ref. } 43 \text {. }\end{array}$ \\
\hline$\theta(\mathrm{X}-\mathrm{C}-\mathrm{X})$ in $\mathrm{CX}_{2} \mathrm{Y}_{2}$ & $\begin{array}{l}108.5^{\circ}, \mathrm{Y}=\mathrm{H} \\
109.5^{\circ}, \mathrm{Y}=\mathrm{Cl}\end{array}$ & $\begin{array}{l}111.8^{\circ}, Y=H \\
108.5^{\circ}, Y=F\end{array}$ & $\begin{array}{l}112.0^{\circ}, \mathrm{Y}=\mathrm{Cl} \\
111.9^{\circ}, \mathrm{Y}=\mathrm{F}\end{array}$ & \\
\hline
\end{tabular}

The parameter values listed in this table are used as a basis for describing in the text various choices of the parameter set.

We use the symbol $\Leftarrow$ to indicate that the $\mu_{j}^{(p)}$ on the right are varied for best (least squares) fit of the expression on the right to that on the left for the set of molecules considered. Equation (19) is similar to Eq. (18) but serves, in this step, to calculate the value of $\hat{\mu}_{i}^{(p)}$ for use in Eq. (18) for the next iteration

$$
\hat{\mu}_{i}^{(p)}=\sum_{j} \mathbf{M}_{i j} \alpha_{j}^{-1}\left(\mu_{j}^{(p)}+\beta_{j}\left\langle\mathrm{~T} \cdot \hat{\mu}^{(p-1)}\right\rangle_{j}^{2}+\gamma_{j}\left(\mathrm{~T} \cdot \hat{\mu}^{(p-1)}\right\rangle_{j}^{3}\right) .
$$

After sufficient iteration the $\hat{\mu}_{i}^{(p)}$ converge to a value $\hat{\mu}_{i}$ and the molecular moment $\mu^{\prime}$ is calculated as

$$
\mu^{\prime}=\sum_{i} \hat{\mu}_{i} .
$$

This algorithm is carried out numerically noting that in Eqs. (18) and (19) $\hat{\mu}_{i}$ is a 12 component vector $\left(\hat{\mu}_{x 1}\right.$, $\left.\hat{\mu}_{y 1}, \cdots, \hat{\mu}_{z 4}\right)$ and $\left(\mathbf{M}_{i j} \alpha_{j}^{-1}\right)$ is a $12 \times 12$ matrix operator in the same space. The number of components can be reduced from 12 to three (for $\mathrm{CXY}_{3}$ type molecules) or four (for $\mathrm{CX}_{2} \mathrm{Y}_{2}$ type molecules) by choice of coordinate systems and by noting that each molecule contains bonds of two types only. For all choices of parameter set (with one exception indicated in Table II) there are no changes greater than $1 \%$ in any variable from the fifth to the sixth iteration of this procedure. This convergence justifies the approach adopted.
The least squares fit of Eq. (18) involves minimizing $\chi^{2}$ (the reduced chi-squared parameter) with respect to $\mu_{i} \cdot \chi^{2}$ is defined by

$$
\chi^{2}=\nu^{-1} \sum_{l}\left(\mu^{\prime}-\mu\right)_{l}^{2} W_{l}^{2},
$$

where the sum is over molecules, $\nu$ is the difference between the number of data points and the number of parameters ( $\nu=6$ here), and $W_{l}$ is a weight. In results quoted explicitly we have used the reciprocals of experimental uncertainties as weights. Arguments can be made for using other weights (all molecules weighted equally, $W_{l}^{-1}$ proportional to $\mu$ for the $l$ th molecule, etc.), but we find that these do not yield qualitatively different fits. The uncertainties listed in parentheses after each $\mu_{i}$ in Table II are as defined by Bevington ${ }^{44}$ and serve mainly to indicate the relative sensitivity of a fit to the various $\mu_{i}$.

The results of fitting with various choices of parameter set are shown in Tables $\amalg-V$. In Table II the full range of parameter-set choices is presented, exhibiting $\mu_{i}$ and $\chi^{2}$ for each. Simple BAA with tetrahedral angles is not included, since only differences between the $\mu_{i}$ can be determined in this case. Use of experimental (nontetrahedral) angles lifts this restriction but the results are sensitive to deviations from tetrahedral sym- 
TABLE II. Bare bond moments $\mu(\mathrm{C}-\mathrm{X})$ obtained by fitting dipole moments of the halogenated methanes. ${ }^{2}$

\begin{tabular}{|c|c|c|c|c|}
\hline Parameter values & $\mu(\mathrm{C}-\mathrm{F})$ & $\mu(\mathrm{C}-\mathrm{Cl})$ & $\mu(\mathrm{C}-\mathrm{H})$ & $x^{2}$ \\
\hline Simple BAA $\left(\alpha_{i}, \beta_{i}, \gamma_{i}=0\right)$; experimental angles ${ }^{b}$ & $4.75(8)$ & $4.54(9)$ & $3.11(9)$ & 109 \\
\hline Isotropic $\alpha_{i} ; \beta_{i}, \gamma_{i}=0$; tetrahedral angles & $1.75(3)$ & $1.84(4)$ & $0.80(4)$ & 31 \\
\hline Isotropic $\alpha_{i}: \beta_{i}, \gamma_{i}=0$; experimental angles & $1.70(3)$ & $1.87(3)$ & $0.74(4)$ & 12 \\
\hline A nisotropic $\alpha_{i} ; \beta_{i}, \gamma_{i}=0$; tetrahedral angles & $1.57(2)$ & $1.80(2)$ & $0.64(3)$ & 12 \\
\hline Anisotropic $\alpha_{i} ; \beta_{i}, \gamma_{i}=0 ;$ experimental angles ${ }^{\mathrm{b}}$ & $1.52(2)$ & $1.76(2)$ & $0.59(2)$ & 8 \\
\hline Isotropic $\alpha_{i} ; \beta_{i}, \gamma_{i} \neq 0$; tetrahedral angles ${ }^{\mathrm{c}}$ & $\cdots$ & $\cdots$ & $\cdots$ & $\cdots$ \\
\hline Isotropic $\alpha_{i} ; \beta_{i} ; \gamma_{i} \neq 0$; experimental angles & $1.65(3)$ & $1.79(4)$ & $0.42(4)$ & 37 \\
\hline Anisotropic $\alpha_{i} ; \beta_{i}, \gamma_{i} \neq 0 ;$ tetrahedral angles & $1.47(2)$ & $1.65(2)$ & $0.34(3)$ & 33 \\
\hline Anisotropic $\alpha_{i} ; \beta_{i}, \gamma_{i} \neq 0$; experimental angles ${ }^{b}$ & $1.40(2)$ & $1.59(2)$ & $0.28(2)$ & 13 \\
\hline
\end{tabular}

The bare bond moments are in debye units $\left(10^{-18}\right.$ esu). The positive sign of $\mu(C-X)$ in all cases implies an electric polarization of the bond in the same $\mathrm{C}^{+}-\mathrm{X}^{-}$.

'The fitted molecular dipole moment $\mu^{\prime}$ for each molecule is listed in Table III.

${ }^{\circ} \mathrm{Calculation}$ failed to converge satisfactorily.

metry. Some variation of the results with choice of parameters may be noted: Firstly, all of the fits in which interactions are included have significantly smaller $\chi^{2}$ than the BAA calculation; and secondly, for both isotropic and anisotropic $\alpha_{i}$ use of experimental bond angles yields a better fit than obtained with tetrahedral angles. It should be emphasized that $\alpha_{i}, \beta_{i}$, and $\gamma_{i}$ have not been varied to minimize $\chi^{2}$.

The values of bare bond dipole moment $\mu(\mathrm{C}-\mathrm{X})$ from Table II can usefully be compared with corresponding results from other sources. Analyses of infrared intensity data ${ }^{45}$ have yielded estimates of $\mu(C-X)$, but the reliability of such estimates based on the bond moment hypothesis have been strongly criticized. ${ }^{46,47}$ Nevertheless, there appears to be a consensus that $\mu(\mathrm{C}-\mathrm{H})$ for $\mathrm{CH}_{4}{ }^{48}$ is approximately $0.35 \mathrm{D}$, in close agreement with the results of Table II for anisotropic $\alpha_{i}$ and $\beta_{i}, \gamma_{i} \neq 0$. Theoretical calculations ${ }^{16,17}$ of $\mu(\mathrm{C}-\mathrm{H})$ confirm that it is positive (i.e., polarized in the sense $\mathrm{C}^{+}-\mathrm{H}^{-}$), but predict a much larger magnitude $(2.0 \mathrm{D})$. Infrared intensity data do not appear to yield physically unambiguous estimates of $\mu(\mathrm{C}-\mathrm{F})$ or $\mu(\mathrm{C}-\mathrm{Cl})$.

In Table III fitted molecular moments $\mu^{\prime}$ are compared with the experimentally determined values $\mu$ for various levels of interaction. All results here are for experimental bond angles and anisotropic $\alpha_{i}$. Results

TABLE III. Experimental and fitted electric dipole moments for halogenated methanes. ${ }^{2}$

\begin{tabular}{|c|c|c|c|c|c|c|c|c|c|}
\hline \multirow[b]{2}{*}{ Molecule } & \multirow[b]{2}{*}{$\mu^{b}$} & \multicolumn{2}{|c|}{$\alpha_{i}, \beta_{i}, \gamma_{i}=0^{c, 8}$} & \multicolumn{2}{|c|}{$\alpha_{i} \neq 0 ; \beta_{i}, \gamma_{i}=0^{g}$} & \multicolumn{2}{|c|}{$\alpha_{i}, \beta_{i}, \gamma_{i} \neq 0^{g}$} & \multicolumn{2}{|c|}{$\begin{array}{c}\text { Smith et al. } \\
\text { (Ref. 21) }\end{array}$} \\
\hline & & $\mu^{\prime}$ & Diff. & $\mu^{\prime}$ & Diff. & $\mu^{\prime}$ & Diff. & $\mu^{\prime d}$ & Diff. \\
\hline $\mathrm{CHCl}_{3}$ & 1. $04(2)$ & 1.10 & -0.06 & 0.98 & +0.06 & 1.14 & -0.10 & 1. 12 & -0.11 \\
\hline $\mathrm{CH}_{2} \mathrm{Cl}_{2}$ & $1.60(3)$ & 1.62 & -0.02 & 1.60 & 0.0 & 1. 64 & -0.04 & 1.63 & -0.06 \\
\hline $\mathrm{CH}_{3} \mathrm{Cl}$ & $1.90(2)$ & 1.66 & +0.24 & 1.89 & +0.01 & 1.88 & +0.02 & $(1.86)^{t}$ & $\cdots$ \\
\hline $\mathrm{CH}_{3} \mathrm{~F}$ & 1. $85(2)$ & 1.75 & +0.10 & 1.91 & -0.06 & 1.91 & -0.06 & $(1.81)^{f}$ & $\cdots$ \\
\hline $\mathrm{CH}_{2} \mathrm{~F}_{2}$ & 1. $98(2)$ & 2.07 & -0.09 & 1.93 & +0.05 & 1.92 & +0.06 & 1.91 & $+0.07^{\mathrm{\theta}}$ \\
\hline $\mathrm{CHF}_{3}$ & $1.65(2)$ & 1.80 & -0.15 & 1.71 & -0.06 & 1.58 & +0.07 & 1.53 & +0.06 \\
\hline $\mathrm{CF}_{3} \mathrm{Cl}$ & $0.50(1)$ & 0.41 & +0.09 & 0.49 & +0.01 & 0.52 & -0.02 & $\cdots$ & $\cdots$ \\
\hline $\mathrm{CF}_{2} \mathrm{Cl}_{2}$ & $0.51(5)$ & 0.18 & +0.33 & 0.45 & +0.06 & 0.48 & +0.03 & 1.18 & -0.67 \\
\hline $\mathrm{CFCl}_{3}$ & $0.46(2)$ & 0.73 & -0.27 & 0.42 & +0.04 & 0.43 & +0.03 & 0.95 & -0.50 \\
\hline
\end{tabular}

'Experimental and fitted dipole moments $\mu$ and $\mu^{\prime}$, respectively, and their differences $\left(\mu-\mu^{\prime}\right)$ are in debye units $\left(10^{-18}\right.$ esu).

${ }^{b}$ Values of $\mu$ are taken from Refs. 23 and $24 . \mu$ is directed along the major symmetry axis and is taken to be in the sense ${ }^{-} \mathrm{F}_{n} \mathrm{CCl}_{4-n}^{+},{ }^{+} \mathrm{H}_{n} \mathrm{CF}_{4-n}^{-}$, or ${ }^{+} \mathrm{H}_{n} \mathrm{CCl}_{4}^{-}$, which is consistent with the experimentally determined ${ }^{49}$ polarizations ${ }^{+} \mathrm{HCF}_{3}^{-}$and ${ }^{+} \mathrm{H}_{3} \mathrm{CCl}^{-}$. Experimental uncertainties are indicated in parentheses.

'The approximation $\alpha_{i}, \beta_{i}, \gamma_{i}=0$ corresponds to the simple BAA situation.

${ }^{d}$ Values of $\mu^{\prime}$ from Kef. 21 are those estimatedby assuming that $\mu(\mathrm{C}-\mathrm{H})=0$. Results with $\mu(\mathrm{C}-\mathrm{H})$

$= \pm 0.3 \mathrm{D}$ are also listed in Ref. 21 , but these do not provide a significantly better fit to $\mu$. All angles in this fitting were assumed to be tetrahedral.

'Differences are calculated using values of $\mu$ cited in Ref. 21 , with the exception of $\mathrm{CH}_{2} \mathrm{~F}_{2}$, for which $\mu$ from Ref. 23 was used.

'The fitting procedure of Ref. 21 constrains $\mu$ ' to equal $\mu$ for $\mathrm{CH}_{3} \mathrm{Cl}$ and $\mathrm{CH}_{3} \mathrm{~F}$.

${ }^{6}$ Experimental bond angles (see Table I) and anisotropic $\alpha_{i}$ were used. 
TABLE IV. Contributions to $\hat{\mu}_{i}$ for $\mathrm{C} . \mathrm{X}$ bonds in halogenated methanes, based on Eq. (10). ${ }^{2}$

\begin{tabular}{|c|c|c|c|c|c|c|c|c|}
\hline \multirow[b]{2}{*}{ Molecule } & \multirow[b]{2}{*}{ Bond $i$} & \multirow[b]{2}{*}{$\mu_{i}^{b}$} & \multicolumn{3}{|c|}{ Components parallel to bond axis } & \multicolumn{3}{|c|}{$\begin{array}{c}\text { Components perpendicular to } \\
\text { bond } \text { axis }^{c}\end{array}$} \\
\hline & & & $\alpha_{i}\langle\mathrm{~T} \cdot \hat{\mu}\rangle_{i}$ & $\boldsymbol{\beta}_{\boldsymbol{i}}\langle\mathrm{T} \cdot \hat{\mu}\rangle_{i}^{2}$ & $\gamma_{i}\langle\mathrm{~T} \cdot \hat{\mu}\rangle_{i}^{3}$ & $\alpha_{i}\langle\mathrm{~T} \cdot \hat{\mu}\rangle_{i}$ & $\beta_{i}\langle\mathbf{T} \cdot \hat{\mu}\rangle_{i}^{2}$ & $\gamma_{i}\langle\mathrm{~T} \cdot \hat{\mu}\rangle_{i}^{3}$ \\
\hline \multirow[t]{2}{*}{$\mathrm{CHCl}_{3}$} & $\mathrm{C}-\mathrm{Cl}$ & 1.593 & -0.534 & 0.004 & -0.001 & 0.024 & -0.001 & 0.0 \\
\hline & $\mathrm{C}-\mathrm{H}$ & 0.281 & -0.438 & 0.074 & -0.008 & 0.0 & 0.0 & 0.0 \\
\hline \multirow{2}{*}{$\mathrm{CH}_{2} \mathrm{Cl}_{2}$} & $\mathrm{C}-\mathrm{Cl}$ & 1.593 & -0.285 & 0.001 & 0.0 & 0.028 & 0.0 & 0.0 \\
\hline & $\mathrm{C}-\mathrm{H}$ & 0.281 & -0.349 & 0.049 & -0.004 & 0.072 & -0.011 & 0.001 \\
\hline \multirow[t]{2}{*}{$\mathrm{CH}_{3} \mathrm{Cl}$} & $\mathrm{C}-\mathrm{Cl}$ & 1.593 & 0.119 & 0.0 & 0.0 & 0.0 & 0.0 & 0.0 \\
\hline & $\mathrm{C}-\mathrm{H}$ & 0.281 & -0.307 & 0.038 & -0.003 & 0.070 & -0.009 & 0.0 \\
\hline \multirow[t]{2}{*}{$\mathrm{CH}_{3} \mathrm{~F}$} & $\mathrm{C}-\mathrm{F}$ & 1.406 & 0.186 & -0.002 & 0.0 & 0.0 & 0.0 & 0.0 \\
\hline & $\mathrm{C}-\mathrm{H}$ & 0.281 & -0.370 & 0.058 & -0.005 & 0.117 & -0.019 & 0.002 \\
\hline \multirow[t]{2}{*}{$\mathrm{CH}_{2} \mathrm{~F}_{2}$} & $\mathrm{C}-\mathrm{F}$ & 1.406 & -0.096 & 0.0 & 0.0 & 0.012 & 0.0 & 0.0 \\
\hline & $\mathrm{C}-\mathrm{H}$ & 0.281 & -0.533 & 0.119 & -0.016 & 0.153 & -0.036 & 0.004 \\
\hline \multirow[t]{2}{*}{$\mathrm{CHF}_{3}$} & $\mathrm{C}-\mathrm{F}$ & 1.406 & -0.301 & 0.004 & 0.0 & 0.011 & 0.001 & 0.0 \\
\hline & $\mathrm{C}-\mathrm{H}$ & 0.281 & -0.962 & 0.360 & -0.085 & 0.0 & 0.0 & 0.0 \\
\hline \multirow[t]{2}{*}{$\mathrm{CF}_{3} \mathrm{Cl}$} & $C-F$ & 1.406 & -0.486 & 0.011 & -0.001 & 0.013 & 0.002 & 0.0 \\
\hline & $\mathrm{C}-\mathrm{Cl}$ & 1.593 & -1.116 & 0.016 & -0.007 & 0.0 & 0.0 & 0.0 \\
\hline \multirow[t]{2}{*}{$\mathrm{CF}_{2} \mathrm{Cl}_{2}$} & $\mathrm{C}-\mathrm{F}$ & 1.406 & -0.397 & 0.008 & 0.0 & 0.011 & 0.001 & 0.0 \\
\hline & $\mathrm{C}-\mathrm{Cl}$ & 1.593 & -0.991 & 0.013 & -0.005 & -0.002 & 0.0 & 0.0 \\
\hline \multirow[t]{2}{*}{$\mathrm{CFCl}_{3}$} & $\mathrm{C}-\mathrm{F}$ & 1. 406 & -0.324 & -0.005 & 0.0 & 0.0 & 0.0 & 0.0 \\
\hline & $\mathrm{C}-\mathrm{C} 1$ & 1. 593 & -0.859 & 0.010 & -0.003 & 0.001 & 0.0 & 0.0 \\
\hline
\end{tabular}

${ }^{2}$ All numerical entries are in debye units and are calculated using anisotopic $\alpha_{i}$ and experimental bond angles. The results correspond to the entries labeled $\alpha_{i}, \beta_{i}, \gamma_{i} \neq 0$ in Table III and to the final row of Table II. Computed results are shown to three places of decimals to exhibit small components. Physically meaningful precision at this level is not implied.

${ }^{6}$ Note that the component of the bare bond moment $\mu_{i}$ perpendicular to the bond axis is identically zero, in contrast to the perpendicular components of the dressed bond moments $\hat{\mu}_{i}$.

'Perpendicular components lie in the plane including the bond and the molecular $z$ axis. A positive sign indicates a positive component along the molecular $+z$ direction.

of Smith et al. ${ }^{21}$ are included for comparison. In addition to having better $\chi^{2}$ the cases with interactions included reproduce the experimentally determined ordering of moment values: This is not the case either with BAA or with the calculation of Smith et al..$^{21}$ It is conceivable that the method of Smith et al ${ }^{21}$ might more closely reproduce the experimental data if its parameters were determined by a least squares fit to all the data, rather than by constraining the fit to just two molecules $\left(\mathrm{CH}_{3} \mathrm{Cl}\right.$ and $\left.\mathrm{CH}_{3} \mathrm{~F}\right)$.

Table IV displays the detailed contributions of the various terms to the dressed moments $\hat{\mu}_{i}$ of the bonds in each of the molecules included in the fit. Of particular interest are the large $\beta_{i}\langle\mathrm{~T} \cdot \hat{\mu}\rangle_{i}^{2}$ contributions parallel to the bond axis in the $\mathrm{C}-\mathrm{H}$ bonds of $\mathrm{CHCl}_{3}, \mathrm{CH}_{2} \mathrm{~F}_{2}$, and $\mathrm{CHF}_{3}$, due to large dipole moments of $\mathrm{C}-\mathrm{Cl}$ and $\mathrm{C}-\mathrm{F}$ bonds acting upon the $\beta_{i}$ of the $\mathrm{C}-\mathrm{H}$ bond. Also, there are large $\alpha_{i}\langle\mathrm{~T} \cdot \hat{\mu}\rangle_{i}$ contributions perpendicular to the bond axes in the $\mathrm{C}-\mathrm{H}$ bonds of $\mathrm{CH}_{3} \mathrm{~F}$ and $\mathrm{CH}_{2} \mathrm{~F}_{2}$. In most cases $\alpha_{i}\langle\mathbf{T} \cdot \hat{\mu}\rangle_{i}$ parallel to the bond axis makes a significant contribution to $\hat{\mu}_{i}$.

Table $\mathrm{V}$ contains fitted molecular moments resolved into contributions arising from various interactions corresponding to the terms in Eq. (10). These data can best be examined in three groups. In the $\mathrm{CH}_{n} \mathrm{Cl}_{4-\pi} \mathrm{mol}-$ ecules $\alpha_{i}\langle T \cdot \hat{\mu}\rangle_{i}$ contributions are considerably smaller than $\mu_{i}$ terms, and $\beta_{i}\langle\mathbf{T} \cdot \hat{\mu}\rangle_{i}^{2}$ terms are fairly small. In
$\mathrm{CH}_{n} \mathrm{~F}_{4-n}$ molecules the $\alpha_{i}\langle\mathrm{~T} \cdot \hat{\mu}\rangle_{i}$ are somewhat larger in relation to $\mu_{i}$, and $\beta_{i}\langle\mathbf{T} \cdot \hat{\mu}\rangle_{i}^{2}$ becomes appreciable. In $\mathrm{CF}_{n} \mathrm{Cl}_{4-\pi} \alpha_{i}\langle\mathrm{~T} \cdot \hat{\mu}\rangle_{t}$ terms overwhelm $\mu_{i}$, and the $\beta_{i}\langle\mathrm{~T}$ $\cdot \hat{\mu}\rangle_{i}^{2}$ terms are small. Looking at results for all nine molecules the largest $\beta_{i}\langle\mathbf{T} \cdot \hat{\mu}\rangle_{i}^{2}$ contribution is about $22 \%$ of $\mu^{\prime}$ and in all other cases it contributes less than $10 \%$. The average $\gamma_{i}\langle\mathbf{T} \cdot \hat{\mu}\rangle_{i}^{3}$ contribution is $1 \%$.

TABLE V. Contributions to the fitted electric dipole moments for halogenated methanes. ${ }^{2}$

\begin{tabular}{llllll}
\hline \hline & & \multicolumn{4}{c}{ Components parallel to molecular $z$ axis $^{\mathrm{b}}$} \\
\cline { 3 - 6 } Molecule & $\mu^{\prime c}$ & $\sum_{i} \mu_{i}$ & $\sum_{i} \alpha_{i}\langle\mathrm{~T} \cdot \hat{\mu}\rangle_{i}$ & $\sum_{i} \beta_{i}\langle\mathrm{~T} \cdot \hat{\mu}\rangle_{i}^{2}$ & $\sum_{i} \gamma_{i}\langle\mathrm{~T} \cdot \hat{\mu}\rangle_{i}^{3}$ \\
\hline $\mathrm{CHCl}_{3}$ & 1.141 & 1.196 & 0.010 & -0.073 & 0.007 \\
$\mathrm{CH}_{2} \mathrm{Cl}_{2}$ & 1.643 & 1.473 & 0.238 & -0.073 & 0.006 \\
$\mathrm{CH}_{3} \mathrm{Cl}$ & 1.877 & 1.333 & 0.602 & -0.062 & 0.004 \\
$\mathrm{CH}_{3} \mathrm{~F}$ & 1.910 & 1.134 & 0.875 & -0.109 & 0.009 \\
$\mathrm{CH}_{2} \mathrm{~F}_{2}$ & 1.918 & 1.328 & 0.757 & -0.192 & 0.024 \\
$\mathrm{CHF}_{3}$ & 1.584 & 1.171 & 0.681 & -0.353 & 0.085 \\
$\mathrm{CF}_{3} \mathrm{Cl}_{1}$ & 0.524 & -0.128 & 0.646 & -0.004 & 0.006 \\
$\mathrm{CF}_{2} \mathrm{Cl}_{2}$ & 0.475 & -0.239 & 0.714 & -0.004 & 0.005 \\
$\mathrm{CFCl}_{3}$ & 0.431 & 0.007 & 0.439 & 0.000 & 0.003 \\
\hline $\mathrm{F}^{2}$ & &
\end{tabular}

a Fitted electric dipole moments $\mu^{\prime}$ and all contributions are in debye units. Computed results are shown to three places of decimals to exhibit small components. Physically meaningful precision at this level is not implied.

${ }^{b}$ Molecular $z$ axis is defined to point in the $\rightarrow^{+}$direction of the molecular dipole moment.

'The parameters used in this calculation are: anisotropic $\alpha_{i}$ $\beta_{i}, \gamma_{i} \neq 0$; experimental bond angles. 
The foregoing results encourage confidence that the ISM approach, in general, and the iterative method of computation, in particular, represent a valid means of understanding the dipole moments of the molecules considered. Moreover, the results are not particularly sensitive to the (poorly determined) values of $\beta_{i}$ and $\gamma_{i}$, which in turn justifies the omission of higher order hyperpolarizability contributions. Uncertainties in $\alpha_{i}$, however, are important, and more reliable data for these bare bond values will be provided by an ISM study $^{33}$ of $\alpha$ analogous to that presented here for $\mu$.

\section{CONCLUSION}

We have developed the theory of an interacting segment model (ISM) of the molecular electric dipole moment, polarizability, and hyperpolarizabilities. A detailed ISM for dipole moments with segments taken to be bonds has been applied to the dipole moments of some halogenated methanes. It has been demonstrated that it is possible to treat terms in $\beta_{i}$ and $\gamma_{i}$ as perturbations and to omit contributions depending on higher order hyperpolarizabilities. The resulting fit has proved to be good and to produce bond dipole moments $\mu(\mathrm{C}-\mathrm{X})$ which are reasonably consistent with expectations from other sources, such as infrared intensity data.

The model is characterized by a number of parameters which are not varied for best fit (see Table I). The choice of values for these parameters involves crude estimates of bare values from measured dressed parameters. It is anticipated that the number of such crudely estimated parameters will be reduced in further computations ${ }^{33}$ in which the fitting procedure will be extended to include ISM calculations for $\alpha_{i}, \beta_{i}$, and $\gamma_{i}$. The quality of this overall fit should give a better measure of the usefulness and limitations of the model than can be obtained from the present preliminary computations.

\section{ACKNOWLEDGMENTS}

It is a pleasure to acknowledge helpful discussions with M. P. Bogaard, B. F. Levine, and W. F. Murphy.

\section{APPENDIX: EXTENSION TO TIME VARYING FIELDS AND DEFINITION OF MOLECULAR OBSERVABLES}

In most experiments used to measure the electric tensor properties $\mu, \alpha, \beta$, and $\gamma$ the applied electric field is time dependent and is of the general form

$$
\mathbf{E}(t)=\mathbf{E}^{0}+\frac{1}{2} \sum_{j}\left[\mathbf{E}^{\omega_{j}} \exp \left(-i \omega_{j} t\right)+\mathbf{E}^{-\omega_{j}} \exp \left(+i \omega_{j} t\right)\right] .
$$

The resultant electric dipole moment can be similarly expressed in terms of Fourier components

$$
\begin{aligned}
& \mu(\mathbf{E}, t)=\mu^{0}(\mathbf{E})+\frac{1}{2} \sum_{\sigma} {\left[\mu^{\omega_{\sigma}}(\mathbf{E}) \exp \left(-i \omega_{\sigma} t\right)\right.} \\
&\left.+\mu^{-\omega_{\sigma}}(\mathbf{E}) \exp \left(+i \omega_{\sigma} t\right)\right],
\end{aligned}
$$

where, for a specific combination $\omega_{1}, \omega_{2}, \ldots, \omega_{n}$ of applied field frequencies which together yield a resultant frequency $\omega_{\sigma}=\omega_{1}+\omega_{2}+\cdots+\omega_{n}$, we can write

$$
\begin{aligned}
\mu^{\omega_{\sigma}}(\mathbf{E})= & \sum_{n=0}^{\infty} K\left(-\omega_{\sigma} ; \omega_{1}, \omega_{2}, \cdots, \omega_{n}\right) \\
& \times \chi^{(n)}\left(-\omega_{0} ; \omega_{1}, \omega_{2}, \cdots, \omega_{n}\right) \mathbf{E}^{\omega_{1}} \mathbf{E}^{\omega_{2}} \cdots \mathbf{E}^{\omega_{n}} .
\end{aligned}
$$

Equation (A3) is a more general form of Eq. (4), which can now describe phenomena with general time dependence. Definition of the numerical factors $K\left(-\omega_{\sigma} ; \omega_{1}\right.$, $\omega_{2}, \ldots$ ) may be completed by specifying

$$
\begin{aligned}
& K(0 ; 0, \cdots, 0)=1, \text { for all } n, \\
& \operatorname{Lim}_{a l l \omega \rightarrow 0} \chi^{(n)}\left(-\omega_{\sigma} ; \omega_{1}, \cdots, \omega_{n}\right)=\chi^{(n)}(0 ; 0, \cdots, 0) .
\end{aligned}
$$

This definition follows the convention previously adopted by Ward and co-workers and numerical values of these $K$ 's for specific combinations of field frequencies have been evaluated and tabulated. ${ }^{2,50}$ It should be noted however that there is a wide variety of different conventions in current use. ${ }^{1,10}$

For dc fields Eq. (A4) states that all $K^{\prime}$ s are unity in this convention and that Eq. (A3) reduces to the form used in Eq. (4) or, in terms of dressed tensors, Eq. (3). Also, by comparison with Eq. (1) it can be seen that the constants $\zeta$ and $\lambda$ used there are unity in this convention.

The derivation in Sec. II leading to the relations between $\hat{\chi}_{i}^{(n)}$ and $\chi_{i}^{(n)}$ set out in Eqs. (10)-(13) can be reworked using the more general time-dependent expressions Eqs. (A1)-(A3) in place of the original expressions relevant to dc fields only. A typical result is the segment hyperpolarizability for dc-induced second-harmonic generation, where Eq. (13) is replaced by a far richer expression containing, among other parameters, polarizabilities for all three frequencies involved. The frequency dependent expression is ${ }^{38}$

$$
\begin{aligned}
\hat{\gamma}_{i}(- & 2 \omega ; \omega, \omega, 0)=\alpha_{i}(-2 \omega ; 2 \omega)\langle\mathrm{T} \cdot \hat{\gamma}(-2 \omega ; \omega, \omega, 0)\rangle_{i}+2 \beta_{i}(-2 \omega ; 2 \omega, 0)\left\{\langle\mathrm{T} \cdot \hat{\mu}\rangle_{i}\langle\mathrm{~T} \cdot \hat{\gamma}(-2 \omega ; \omega, \omega, 0)\rangle_{i}\right. \\
& \left.+\frac{1}{3}\left[1+\langle\mathrm{T} \cdot \hat{\alpha}(0 ; 0)\rangle_{i}\right]\langle\mathrm{T} \cdot \hat{\beta}(-2 \omega ; \omega, \omega)\rangle_{i}\right\}+\frac{4}{3} \beta_{i}(-2 \omega ; \omega, \omega)\left[1+\langle\mathrm{T} \cdot \hat{\alpha}(-\omega ; \omega)\rangle_{i}\right]\langle\mathrm{T} \cdot \hat{\beta}(-\omega ; \omega, 0)\rangle_{i} \\
& +\gamma_{i}(-2 \omega ; 2 \omega, 0,0)\left\{3\langle\mathrm{~T} \cdot \hat{\mu}\rangle_{i}^{2}\langle\mathrm{~T} \cdot \hat{\gamma}(-2 \omega ; \omega, \omega, 0)\rangle_{i}+2\langle\mathrm{~T} \cdot \hat{\mu}\rangle_{i}\left[1+\langle\mathrm{T} \cdot \hat{\alpha}(0 ; 0)\rangle_{i}\right]\langle\mathrm{T} \cdot \hat{\beta}(-2 \omega ; \omega, \omega)\rangle_{i}\right\} \\
& +\gamma_{i}(-2 \omega ; \omega, \omega, \hat{0})\left\{4\langle\mathrm{~T} \cdot \hat{\mu}\rangle_{i}\left[1+\langle\mathrm{T} \cdot \hat{\alpha}(-\omega ; \omega)\rangle_{i}\right]\langle\mathrm{T} \cdot \hat{\beta}(-\omega ; \omega, 0)\rangle_{i}+\left[1+\langle\mathrm{T} \cdot \hat{\alpha}(-\omega ; \omega)\rangle_{i}\right]^{2}\left[1+\langle\mathrm{T} \cdot \hat{\alpha}(0 ; 0)\rangle_{i}\right]\right\}
\end{aligned}
$$

Although polarizabilities and hyperpolarizabilities $\chi^{(n)}$ of a given order $n$ and for a given molecule vary appreciably as a result of dispersion, such variations are in many cases negligible when compared to the large nonadditive contributions from intersegment interactions which vary greatly from molecule to molecule and which 
TABLE VI. Definition of observable molecular electric tensor properties.

\begin{tabular}{|c|c|c|c|}
\hline Electric tensor & Molecular observable $2, b$ & Experimental source $(s)^{c}$ & Comments \\
\hline$\mu$ & $\mu=\left|\left(\mu_{\xi} \mu_{\xi}\right)^{1 / 2}\right|$ & $\begin{array}{l}\text { Microwave spectra } \\
\text { Dielectric constant }\end{array}$ & We choose the molecular $z$ axis such that $\mu=\mu_{z}$. \\
\hline $\begin{array}{l}\alpha(0 ; 0) \\
\quad=\alpha^{0}\end{array}$ & $\alpha^{0}=\frac{1}{3} \alpha_{\mathrm{Gl}}^{0}=\frac{1}{3} \operatorname{Tr}\left(\alpha^{0}\right)$ & Dielectric constant & $\alpha^{0}$ is the isotropic part of $\alpha^{0}$. \\
\hline \multirow{3}{*}{$\begin{array}{l}\alpha(-\omega ; \omega) \\
\quad=\alpha^{\omega}\end{array}$} & $\alpha^{\omega}=\frac{1}{3} \alpha_{\zeta \zeta}^{\omega}=\frac{1}{3} \operatorname{Tr}\left(\alpha^{\omega}\right)$ & Refractive index & $\alpha^{\omega}$ is the isotropic part of $\alpha^{\omega}$. \\
\hline & $\begin{aligned} \Delta \alpha & =3 \alpha^{\omega}{ }_{K} \\
& =\left\{3\left[\alpha_{\xi \eta}^{\omega} \alpha_{\xi \eta}^{\omega}-3\left(\alpha^{\omega}\right)^{2}\right] / 2\right\}^{1 / 2}\end{aligned}$ & $\begin{array}{l}\text { Depolarization ratio } \\
\quad \text { for Rayleigh scattering }\end{array}$ & $\begin{array}{l}\kappa^{2}=\left[\left(\alpha_{x x}^{\omega}-\alpha_{y y}^{\omega}\right)^{2}+\left(\alpha_{y y}^{\omega}-\alpha_{x x}^{\omega}\right)^{2}+\left(\alpha_{k x}^{\omega}-\alpha_{x x}^{\omega}\right)^{2}\right] / 9\left(\alpha^{\omega}\right)^{2} \\
\text { If molecule has three or higher-fold rotational } \\
\text { symmetry, } \kappa=\left(\alpha_{\|}^{\omega}-\alpha_{\perp}^{\omega}\right) / 3 \alpha^{\omega} \text {, so that } \\
\quad \Delta \alpha=\alpha_{\|}^{\omega}-\alpha_{\perp}^{\omega} .\end{array}$ \\
\hline & $\left(\alpha_{z z}^{\omega}-\alpha^{\omega}\right)$ & $\begin{array}{l}\text { Kerr effect (electric } \\
\text { birefringence) }\end{array}$ & $\begin{array}{l}\text { If molecule has three or higher-fold rotational } \\
\text { symmetry, }\left(\alpha_{k z}^{\omega}-\alpha^{\omega}\right)=\frac{2}{3}\left(\alpha_{11}^{\omega}-\alpha_{1}^{\omega}\right)=\frac{2}{3} \Delta \alpha\end{array}$ \\
\hline $\begin{array}{l}\beta(-2 \omega ; \omega, \omega) \\
\quad=\beta^{\text {SHG }}\end{array}$ & $\begin{aligned} \beta^{\mathrm{SHG}} & =\frac{2}{3} \beta_{\xi \zeta x}(-2 \omega: \omega, \omega) \\
& +\frac{1}{5} \beta_{\text {Et5 }}(-2 \omega ; \omega, \omega)\end{aligned}$ & $\begin{array}{l}\text { dc-induced second } \\
\text { harmonic generation }\end{array}$ & $\begin{array}{l}\text { A second observable, } \beta_{\perp}^{\mathrm{SHG}}=\frac{6}{5} \beta_{z \zeta 5}(-2 \omega ; \omega, \omega) \\
-\frac{3}{5} \beta_{\xi \xi e}(-2 \omega ; \omega, \omega) \text { can be determined by hav- } \\
\text { ing. } E^{\omega} \text { polarized perpendicular to } E^{0} \text {. Usually, } \\
\beta^{\text {SHG }} \text { and } \beta_{\perp}^{\text {sHG }} \text { differ by less than } 5 \% .\end{array}$ \\
\hline $\begin{array}{l}\beta(-\omega ; \omega, 0) \\
\quad=\beta^{K}\end{array}$ & $\begin{aligned} \beta^{K} & =\frac{9}{10} \beta_{\xi \mathbb{K}^{2}}(-\omega ; \omega, 0) \\
& -\frac{3}{10} \beta_{\xi \zeta \varepsilon}(-\omega ; \omega, 0)\end{aligned}$ & $\begin{array}{l}\text { Kerr effect (electric } \\
\text { birefringence) }\end{array}$ & \\
\hline $\begin{array}{l}\gamma(-2 \omega ; \omega, \omega, 0) \\
\quad=\gamma^{E S H G}\end{array}$ & $\begin{aligned} \gamma^{\mathrm{ESHG}} & =\frac{2}{15} \gamma_{5 ! \eta \eta}(-2 \omega ; \omega, \omega, 0) \\
& +\frac{1}{15} \gamma_{5 \eta \pi !}(-2 \omega ; \omega, \omega, 0)\end{aligned}$ & $\begin{array}{l}\text { de-induced second } \\
\text { harmonic generation }\end{array}$ & 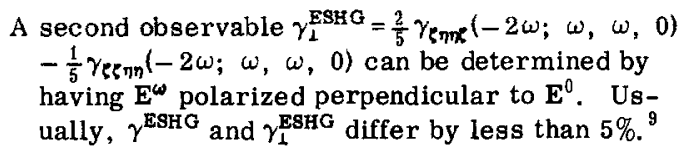 \\
\hline $\begin{array}{l}\gamma(-\omega ; \omega, 0,0) \\
\quad=\gamma^{K}\end{array}$ & $\begin{aligned} \gamma^{K} & =\frac{3}{10} \gamma_{5 \pi \eta_{\eta}}(-\omega ; \omega, 0,0) \\
& -\frac{1}{10} \gamma_{55 \eta \eta}(-\omega ; \omega, 0,0)\end{aligned}$ & $\begin{array}{l}\text { Kerr effect (electric } \\
\text { birefringence) }\end{array}$ & \\
\hline $\begin{array}{l}\gamma(-3 \omega ; \omega, \omega, \omega) \\
=\gamma^{\mathrm{THG}}\end{array}$ & $\gamma^{\mathrm{THG}}=\frac{1}{5} \gamma_{\xi \xi \eta \eta}(-3 \omega ; \omega, \omega, \omega)$ & Third harmonic generation & \\
\hline
\end{tabular}

aCartesian tensor notation ${ }^{36}$ is used where convenient.

${ }^{\mathrm{b}}$ Axes $x, y, z$ are principal molecular axes, the $z$-axis being specified in the definition of $\mu$.

form the subject of this paper. For example, the chloro- and fluoromethanes do not have electronic absorption bands above $200 \mathrm{~nm}$, so that the differences due to dispersion between pairs of electric tensor properties, such as $\alpha(0 ; 0)$ and $\alpha(-\omega ; \omega), \beta(-2 \omega ; \omega, \omega)$ and $\beta(-\omega ; \omega, 0)$, and $\gamma(-2 \omega ; \omega, \omega, 0)$ and $\gamma(-\omega ; \omega, 0,0)$, where $\omega$ corresponds to wavelengths above $600 \mathrm{~nm}$, are expected to be relatively small.

The hyperpolarizabilities may be expanded as follows:

$$
\begin{aligned}
& \chi_{i}^{(n)}\left(-\omega_{0} ; \omega_{1}, \cdots, \omega_{l}, \cdots, \omega_{n}\right) \\
& \quad=\operatorname{Lim}_{a l l \omega \rightarrow 0} \chi_{i}^{(n)}\left(-\omega_{0} ; \omega_{1}, \cdots, \omega_{l}, \cdots, \omega_{n}\right) \\
& \quad+\sum_{l} \omega_{l} \operatorname{Lim}_{a l l \omega \rightarrow 0} \frac{\partial}{\partial \omega_{l}}\left[\chi_{i}^{(n)}\left(-\omega_{0} ; \omega_{1}, \cdots, \omega_{l}, \cdots, \omega_{n}\right)\right]+\ldots .
\end{aligned}
$$

As discussed above it is a reasonable approximation to adopt the dispersionless limit by dropping all but the first term on the right of Eq. (A7). Equation (A5) then indicates that, in the present convention, all hyperpolarizabilities of a given order become equal. The frequency labels may therefore be dropped from Eq. (A6), which reduces directly to Eq. (13). It follows that Eqs. $(10)-(13)$ in the body of the text apply in the dispersion-
'See Ref. 1 for a survey of experimental techniques. $\mathrm{d}_{\beta} \mathrm{SHG}^{\mathrm{HG}}$ and $\gamma^{\mathrm{ESHG}}$ are determined with $\mathrm{E}^{\omega}$ polarized parallel to $\mathrm{E}^{0}$.

less limit to hyperpolarizabilities involving time-dependent fields so long as the hyperpolarizabilities are defined in accordance with Eqs. (A1)-(A5).

It is also relevant in this Appendix to define a number of observable linear combinations of electric tensor elements. These definitions are given in Table VI.

${ }^{1}$ For a recent review of much of the relevant literature see $M$. P. Bogaard and B. J. Orr, International Review of Science. Physical Chemistry, edited by A. D. Buckingham (Butterworths, London, 1975), Ser. 2, Vol. 2, p. 149.

${ }^{2}$ B. J. Orr and J. F. Ward, Mol. Phys, 20, 513 (1971).

${ }^{3} \mathrm{~J}$. W. Smith, Electric Dipole Moments (Butterworths, London, 1955).

${ }^{4}$ A. I. Vogel et al. in a series of 52 papers published in $J$. Chem. Soc. between 1934 and 1966. The last paper in the series is J. Chem. Soc. B 1966, 1080.

${ }^{5}$ R. J. W. LeFèvre, Advances in Physical Organic Chemistry, edited by V. Gold (Academic, New York, 1965), Vol. 3, p. 1.

${ }^{6}$ A. D. Buckingham and B. J. Orr, Q. Rev. Chem. Soc. (London) 21, 195 (1967). Note that errors in Eqs. (9)-(11) have been corrected in Ref. 7 .

${ }^{7}$ A. D. Buckingham and B. J. Orr, Trans. Faraday Soc. 68, 673 (1969).

${ }^{8}$ R. S, Finn and J. F. Ward, J. Chem. Phys, 60, 454 (1974).

${ }^{9}$ J. F. Ward and I. J. Bigio, Phys. Rev. A 11, 60 (1975). 
${ }^{10}$ B. F. Levine and C. G. Bethea, J. Chem. Phys. 63, 2666 (1975), and references cited therein.

${ }^{11} \mathrm{~K}$. S. Pitzer, Advances in Chemical Physics, edited by I. Prigogine (Interscience, New York, 1959), Vol. 2, p. 59.

${ }^{12}$ R. L. Kowell and R. S. Stein, J. Chem. Phys. 47, 2985 (1967).

${ }^{13}$ E. M. Mortensen, J. Chem. Phys. 49, 3732 (1968).

${ }^{14} \mathrm{~J}$. Applequist, J. R. Carl, and K. -K. Fung, J. Am. Chem. Soc. 84, 2952 (1972)

${ }^{15}$ A. D. Buckingham and H. Sutter, J. Chem. Phys, 64, 364 (1976).

${ }^{16}$ R. H. Pritchard and C. W. Kern, J. Am. Chem. Soc. 91, 1631 (1969).

${ }^{17}$ M. S. Gordon and W. England, J. Am. Chem. Soc. 94, 5168 (1972).

${ }^{18}$ A. T. Amos and R. J. Crispin, J. Chem. Phys. 63, 1890 (1975); Mol. Phys. 31, 147 (1976).

${ }^{19} \mathrm{~B}$. F. Levine (private communication).

${ }^{20}$ C. P. Smyth and K. B. McAlpine, J. Chem. Phys. 1, 190 (1933).

${ }^{21}$ R. P. Smith, T. Ree, J. L. Magee, and H. Eyring, J. Am. Chem. Soc. 73, 2263 (1951).

${ }^{22}$ See also the Appendix.

${ }^{23}$ Landolt-Börnstein, Zahlenwerte und Funktionen, Neue Serie, Gruppe II, Atom- und Molekularphysik (Springer, Berlin, 1974), Ser. 6.

${ }^{24}$ R. D. Nelson, D. R. Lide, and A. A. Maryott, Selected Values of Electric Dipole Moments for Molecules in the Gas Phase (U.S. Department of Commerce, Washington, 1967).

${ }^{25}$ K. L. Ramaswamy, Proc. Indian Acad. Sci. Sect. A 4, 675 (1936).

${ }^{26} \mathrm{H}$. E. Watson and K. L. Ramaswamy, Proc. R. Soc. (London) Ser. A 156, 144 (1936).

${ }^{27}$ K. L. Ramaswamy, Proc. Indian Acad. Sci. Sect. A 2, 630 (1935).

${ }^{28} \mathrm{H}$. Sutter and R. H. Cole, J. Chem. Phys. 62, 132 (1970).

${ }^{29}$ T. K. Bose, J. S. Sochanski, and R. H. Cole, J. Chem. Phys. 57, 3592 (1972).

${ }^{30}$ Landolt-Börnstein, Zahlenwerte und Funktionen (Springer, Berlin, 1951), Ser. I, p. 515.

${ }^{31}$ N. J. Bridge and A. D. Buckingham, Proc. R. Soc. (London) Ser. A 295, 334 (1966).

${ }^{32}$ C. K. Miller and J. F. Ward, Phys. Rev. A (to be published).

${ }^{33} \mathrm{C}$. K. Miller, B. J. Orr, and J. F. Ward (to be published).
${ }^{34}$ K. R. Sundberg, J. Chem. Phys. 66, 114, 1475 (1977).

${ }^{35} \mathrm{~J}$. O. Hirschfelder, C. F. Curtiss, and R. B. Bird, Molecular Theory of Gases and Liquids (Wiley, New York, 1954).

${ }^{36} \mathrm{We}$ use conventional Cartesian tensor notation, in which Greek suffixes denote $X, Y, Z$ components and in which summation over those components is understood when the same Greek suffix is repeated

${ }^{37}$ See, for example, A. D. Buckingham, Discuss. Faraday Soc. 40, 232 (1965); Advances in Chemical Physics, edited by J. O. Hirschfelder (Interscience, New York, 1967), Vol. 12 , p. 107.

${ }^{38} \mathrm{We}$ use the shorthand notation $\langle\mathrm{T} \cdot \hat{\chi}\rangle_{i}$ to represent summations of the form $\sum_{j \neq i} T_{i j} \hat{x}_{j}$, where $j$ is a dummy suffix. The notation $\langle\mathbf{T} \cdot \hat{\chi}\rangle_{i}^{s}$ specifies a product of $s$ such terms, each involving summation over a distinct dummy suffix.

${ }^{39} \mathrm{It}$ should be noted that we define our interaction tensors $\mathrm{T}_{i j}$ with opposite sign to that adpoted by Applequist et al ${ }^{14}$ Our matrix $M_{i j}$ is otherwise identical to their matrix $B_{i j}$.

${ }^{40} \mathrm{~L}$. Silberstein, Philos. Mag. 33, 92, 215, 521 (1917).

${ }^{41}$ H. A. Stuart, Molekülstruktur (Springer, Berlin, 1934), p. 184.

${ }^{42}$ W. F. Murphy, W. Holzer, and H. J. Bernstein, Appl. Spectrosc. 23, 211 (1969).

${ }^{43} \mathrm{~L}$. E. Sutton et al., Tables of Interatomic Distances and Configuration in Molecules and Ions (Chemical Society, London, 1958); Interatomic Distances Supplement (Chemical Society, London, 1965).

${ }^{44} \mathrm{P}$. R. Bevington, Data Reduction and Error Analysis for the Physical Sciences (McGraw-Hill, New York, 1969), pp. 243, 188.

${ }^{45} \mathrm{~A}$ recent review is given by $\mathrm{W}, \mathrm{B}$. Person and $\mathrm{D}$. Steele, Molecular Spectroscopy edited by R. F. Barrow, D. A. Long, and D. J. Millen (Chemical Society, London, 1974), Vol. 2, p. 357.

${ }^{46}$ D. F. Hornig and D. C. McKean, J. Phys. Chem. 58, 1133 (1955).

${ }^{47}$ D. Steele, Q. Rev. Chem. Soc. 18, 21 (1964).

${ }^{48}$ I. M. Mills, Mol. Phys. 1, $107(1958)$; R. E. Hiller and J. W. Straley, J. Mol. Spectrosc. 5, 24 (1960).

${ }^{49}$ D. van der Hart and W. H. Flygare, Mol. Phys. 18, 77 (1970); W. H. Flygare and R. C. Benson, Mol. Phys. 20, 225 (1971).

${ }^{50}$ In Refs. 1 and $2 K(0 ; 0, \omega,-\omega)$ is erroneously given as $\frac{3}{8}$; the correct value is $\frac{3}{2}$. 